Muséologies

Les cahiers d'études supérieures

muséologies

\title{
Le design au secours de la médiation ? Un dispositif contemporain de médiation en questions
}

\section{Luc Dall'Armellina}

Volume 7, numéro 1, 2014

Le dialogue dans les musées d'art contemporain

URI : https://id.erudit.org/iderudit/1026649ar

DOI : https://doi.org/10.7202/1026649ar

Aller au sommaire du numéro

Éditeur(s)

Association Québécoise de Promotion des Recherches Étudiantes en

Muséologie (AQPREM)

ISSN

1718-5181 (imprimé)

1929-7815 (numérique)

Découvrir la revue

Citer cet article

Dall'Armellina, L. (2014). Le design au secours de la médiation ? Un dispositif contemporain de médiation en questions. Muséologies, 7(1), 103-120.

https://doi.org/10.7202/1026649ar

Tous droits réservés (C Association Québécoise de Promotion des Recherches Étudiantes en Muséologie (AQPREM), 2014
Ce document est protégé par la loi sur le droit d'auteur. L'utilisation des services d'Érudit (y compris la reproduction) est assujettie à sa politique d'utilisation que vous pouvez consulter en ligne.

https://apropos.erudit.org/fr/usagers/politique-dutilisation/ 
Article quatre

\section{Le design au secours de la médiation? Un dispositif contemporain de médiation en questions ${ }^{1}$}

Luc Dall'Armellina

"[O]n pourrait dire qu'il y a de la nature humaine dans l'être technique." - Gilbert Simondon, Du mode d'existence des objets techniques (p. 248). 
Luc Dall'Armellina a enseigné les arts et le design des hypermédias à l'École supérieure d'art et de design de Grenoble-Valence (1999-2012); il est intervenu en écoles d'art et de design (Nantes, Orléans, Amiens), d'ingénieurs (Université de technologie de Compiègne), ainsi qu'à l'université (Paris 8, Paris 13). Maître de conférences en arts à l'École supérieure du professorat et de l'éducation (ESPE) de l'Université Cergy-Pontoise depuis 2011, il y enseigne le design et les humanités numériques. Membre du laboratoire EMA (Écoles, Mutations, Apprentissages - EA 4507), il y anime le groupe de recherche ECARTS (Écoles, Cultures, Arts: patrimonialisation, médiation et autres transmissions), partenaire de recherche du musée du Louvre et de la Bibliothèque nationale de France dans le cadre du Labex Patrima (2013-2017). Ses recherches portent sur les mutations esthétiques du faire ensemble dans les dispositifs innovants de médiation artistique et culturelle. lucdall@free.fr 
Dans cet article, nous commencerons par porter un regard sur les approches contemporaines en médiation culturelle pour détailler ensuite celle que nous appellerons de l'art par l'art, c'est-à-dire basée non pas centralement sur un savoir, mais sur une expérience sensible proposée au visiteur par des artistes, avec des médiateurs, dans le cadre d'une activité d'atelier ou de performance.

Nous la prolongerons par la description d'une approche originale de médiation de l'œuvre d'art par l'objet de design, proposée et coordonnée par Joël Paubel ${ }^{2}$ et le service Éducation du musée du Louvre en 2013 dans le cadre du dispositif annuel de médiation des JOP ou «Les jeunes ont la parole $n^{3}$.

Nous interrogerons ici deux objets de médiation par l'objet de design parmi la centaine de ceux qui ont été expérimentés au Louvre durant la saison 2012-2013. Nous enchaînerons avec les descriptions et l'analyse et esquisserons, dans ce mouvement, une réflexion à poursuivre ailleurs, car cet article n'y suffira pas - sur la méthodologie d'évaluation analytique et critique la plus adéquate pour en rendre compte. Notre ambition est que ces deux objets soient décrits et que les termes du choix méthodologique soient posés.

L'hybridation du dispositif œuvre d'art / objet de design / médiateur culturel trouve-t-elle à s'inscrire au niveau des rapports des arts et des techniques, de l'esthétique et de la cognition, de l'individuel et du collectif? Cette hybridation confère au dispositif un caractère singulier; nous évaluerons les enjeux de l'approche méthodologique de la médiologie ${ }^{4}$ et, dans sa continuité, de celle de la sociologie de la traduction (dite aussi ANT pour actor-network theory). Née des travaux de l'école des Mines ParisTech avec Bruno Latour, Michel Callon et Madeleine Akrich à partir de l'étude des conditions de production de la science, cette approche s'inscrit dans le projet d'une anthropologie symétrique ${ }^{5}$ dans laquelle tous les éléments d'une enquête - d'un réseau - sont analysés sans hiérarchie. C'est d'une approche écosystémique que relève l'ANT: œuvres, objets, récits, discours, institutions, tous font réseau, en collectif, humains comme non-humains participent d'une pratique, d'une production et, parfois, d'une innovation.

\section{Des limites de la médiation par les savoirs}

La médiation dont nous ${ }^{6}$ parlerons ici est une pratique d'accompagnement culturel des publics dans l'exercice d'une expérience esthétique et critique avec les œuvres patrimoniales au musée. Elle vise en premier lieu à proposer et à maintenir les conditions d'une rencontre de qualité avec des œuvres qui demandent toujours l'espace et le temps de l'attention pour être goûtées. Elle vise en second lieu à assurer les conditions de possibilité d'une expérience

religion, une doctrine, un genre artistique, une discipline, etc.), une forme d'organisation collective (une église, un parti, une école, une académie) et un système technique de communication (saisie, archivage et circulation des traces). Ou, plus simplement, quand on met en ligne un dire, la façon de le dire et qui tient à le redire. " Source: revue Médiologie. <http:// www.mediologie.org> (consulté le 5 juin 2013).

5 Pour une approche introductive, on pourra se reporter à GROSSETI, Michel. Les limites de la symétrie. À propos de l'ouvrage de Bruno Latour Changer de société. Refaire de la Sociologie. Paris: La Découverte, 2006. <http://sociologies. revues.org/712> (consulté le 5 juin 2013).

6 Je n'ai guère l'habitude d'utiliser la forme académique du " nous ", rédigeant la plupart du temps dans une forme neutre. Ce " nous " fait ici une place particulière à Joël Paubel à l'origine de ce dispositif de médiation par le design. Je reste bien sûr seul responsable de ce que j'écris. 
esthétique pour un public curieux, mais pressé, qui peine, en visite non médiée, à accorder plus de dix secondes par œuvre en moyenne ${ }^{7}$.

Autant dire qu'avec une telle définition la médiation culturelle s'invente une place difficile, entre l'incontournable habitus moderne des industries culturelles (attention flottante, papillonnage, consommation) et le risque constant d'une confiscation de l'expérience sensible du visiteur avec l'œuvre par un discours de médiation trop bavard et souvent immédiat.

La médiation, quand elle sait éviter ces écueils, s'assure l'indépendance qui lui permet d'inventer un espace qui ne soit pas seulement de guidage standardisé et de prescription, de pédagogie et de savoirs, mais qui permette au visiteur d'établir une relation créative avec l'œuvre. La médiation pourra chercher à entrainer chacun à user de sa raison sensible $e^{8}$, dans une expérience fondatrice - c'est notre hypothèse - de production de sa subjectivité et, conséquemment, de création de lien social.

La médiation comme approche pédagogique massifiée des arts a vécu et doit être déconstruite, réinventée; c'est une idée qui traverse la pensée de nombre d'acteurs contemporains de la médiation ${ }^{9}$. Nous avons semble-t-il éprouvé les limites d'un modèle ayant privilégié l'accès ${ }^{10}$ aux biens culturels. Penser qu'il soit possible de régler la question de l'expérience esthétique et de la dimension politique

7 Voir l'illustration 1. La question du déficit d'attention avec laquelle les musées doivent composer fait l'objet de l'œuvre interactive Tape Recorders (Museum of Contemporary Art [MCA], Sydney, 2011) de Rafael Lozano-Hemmer, qui met en scène la durée de présence - littéralement mesurée - du regardeur d'une œuvre au musée. Notons que le dispositif n'y mesure pas l'attention, mais le temps qui lui est (potentiellement) consacré. <http://vimeo.com/34533540> (consulté le 18 décembre 2012).

8 BAUMGARTEN, Alexander Gottliem (dans Esthetica, impens. I.C. Kleyb, 1750) définit l'esthétique comme « science de la connaissance sensible $»$. Il signera l'acte de naissance de cette nouvelle discipline philosophique.

9 On pourra se reporter à SAADA, Serge. Et si on partageait la culture? Essai sur la médiation culturelle et le potentiel du spectateur. Toulouse: L'Attribut, coll. « La culture en questions ", 2011; et CAUNE, Jean. La démocratisation culturelle. Une médiation à bout de souffle. Grenoble: Presses universitaires de de l'art par la simple ouverture massifiée d'un accès au patrimoine soutenu par la pédagogie, c'était museler tant l'art que l'esthétique et, finalement, les invalider, tout en instrumentalisant la pédagogie. Un bilan peu engageant pourrions-nous dire, mais il est aussi possible d'y voir le signe de la vitalité critique qui traverse la médiation dans son processus de transformation.

Sans dispositifs créatifs d'appropriation des œuvres, la subjectivité du visiteur ne peut s'élaborer, car elle est moins de l'ordre d'un savoir à acquérir que d'une (co)naissance, se réalisant souvent dans l'expérience partagée d'un petit groupe. Les modalités du vivre ensemble ont été profondément bouleversées depuis vingt ans et il était nécessaire que la médiation se repositionne. «L'art doit être redéfini en fonction de sa contribution à construire le soi ", écrivait Jean Caune ${ }^{11}$ en 2006 dans un livre de bilan sans concession sur la médiation. Sans vouloir comme lui "redéfinir l'art ", nous proposerions, à sa suite, que la médiation devrait aujourd'hui accueillir avec attention les pouvoirs esthétiques, les capacités symboliques et le potentiel subversif des arts pour créer les conditions de leur appropriation intersubjective, à l'école comme à l'université, au travail comme au musée, par les citoyens de tout âge, sexe et condition. Voilà qui pourrait constituer les bases de nouvelles pratiques artistiques à l'école et en formation tout au long de la vie, tout un programme, aux dimensions esthétiques et

Grenoble, coll. " Art, culture, publics ", 2006; ou encore au catalogue du colloque éponyme (collectif: COUBETERGUES, Philippe, Margaret PFENNINGER, Roland RECHT et Muriel RYNGAERT. L'art peut-il se passer de commentaire(s)? Vitry-sur-Seine: MAC/VAL, coll. "Colloque-Événement ", 2006).

10 Précisons ici que nous centrons notre critique d'une politique tournée vers le simple accès aux œuvres sans en envisager l'accompagnement exigeant. En outre, notre critique de l'accès n'oblitère en rien l'importance de la gratuité d'accès aux œuvres pour tous. Voir par exemple sur ce sujet: HASQUENOPH, Bernard. Le rapport caché sur la gratuité des musées. 2009. <http://www.louvrepourtous.fr/Le-rapportcache-sur-la-gratuite, $247 . \mathrm{html}>$; ou encore: TOBELEM, Jean-Michel. La gratuité des musées. 2009. <http://www. louvrepourtous.fr/La-gratuite-des-musees-vue-par,241. html> (consultés le 10 janvier 2012).

11 CAUNE, Jean, La démocratisation culturelle..., op. cit. 


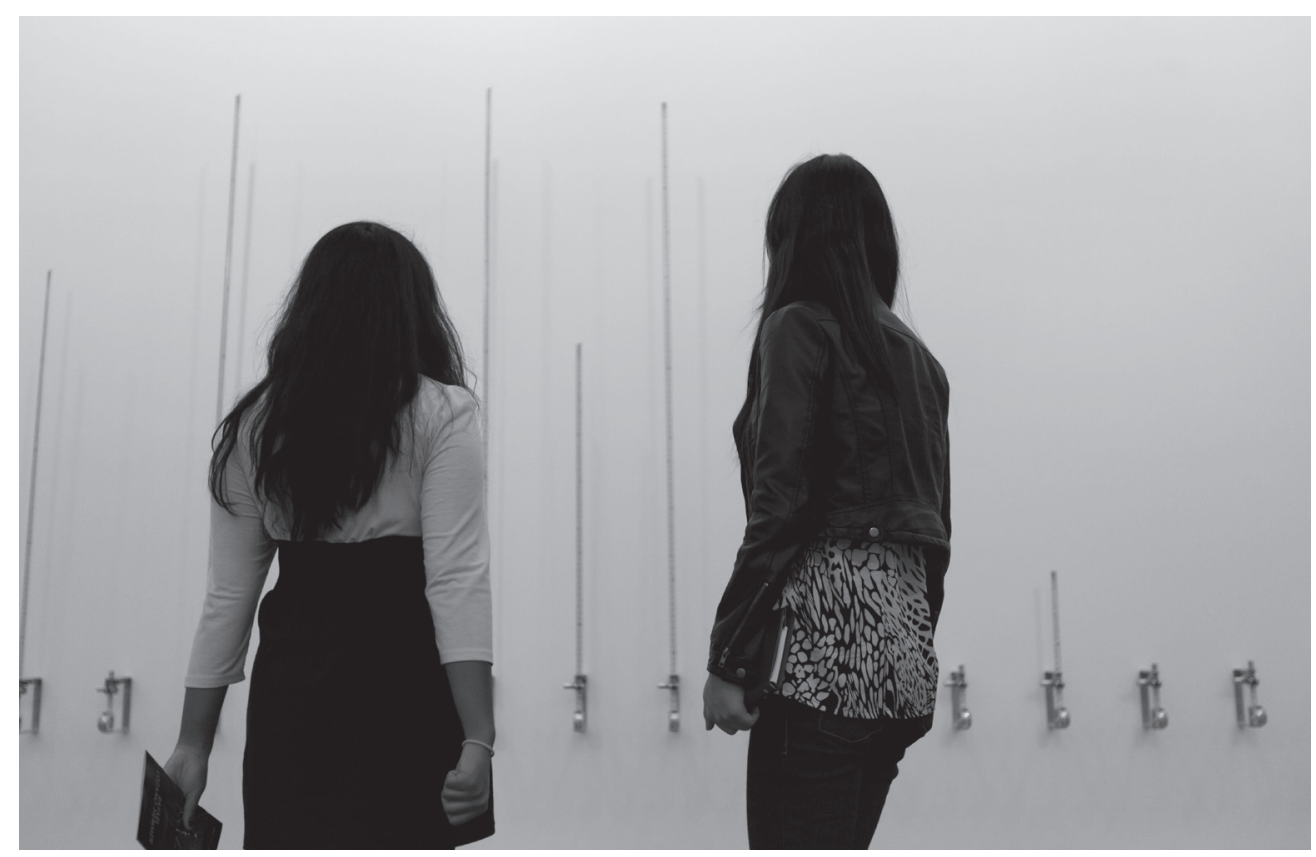

Illustration 1

Rafael Lozano-Hemmer, « Tape Recorders, Subsculpture 12 ", 2011.Recorders, Museum of Contemporary Art, Sydney, Australia.

[Photo: Alex Davies. license CC BY-NC-SA 3.0 ES] 
politiques, que Jacques Rancière a nommé le partage du sensible ${ }^{12}$. La production de l'intersubjectivité - à la base de l'amitié, de la coopération, de l'éthique - ne peut faire défaut trop longtemps sans créer de dommages à la qualité des tissus qui nous lient. La médiation, accompagnatrice bienveillante, parfois naïve et instrumentalisée des industries culturelles, a vécu. Elle se transforme, se réinvente aujourd'hui dans une conscience beaucoup plus fine du contexte dans lequel elle intervient et s'interroge sur les conséquences de ses actions. Nous retrouvons ici la critique de Jean Caune dans une subtile définition du rôle de la médiation à travers laquelle il signalait l'un de ses enjeux majeurs:

La médiation culturelle est processus du temps présent. Qu'elle se présente à partir d'une énonciation singulière ou en interlocution à la parole de l'autre, elle doit laisser, à chaque instant, la possibilité d'une faille qui autorise l'émergence de l'innovation, ou de la trouvaille. Par elle, entre autres, l'avenir pourrait bien ne pas devenir un temps homogène et vide. La fonction de la médiation de la culture consisterait alors moins à faire advenir l'avenir ou à l'annoncer que de maintenir le contact entre hier et aujourd'hui ${ }^{13}$.

Monique Sicard rappelle que ce " contact ", le musée l'intègre en son sein en organisant " un emboîtement de temporalités différentes: celle du bâtiment, celle de la muséographie, celle des objets $"{ }^{14}$. Ce contact entre les temps d'hier et d'aujourd'hui, il semble important de le maintenir, mais plus crucialement encore de s'en saisir. "S'en saisir ", c'est, selon Giorgio Agamben, la question même du contemporain. Etre contemporain, écrit-il, est essentiellement une affaire de courage, et non de temporalité.

12 RANCIÈRE, Jacques, Le partage du sensible, esthétique et politique, Paris: La Fabrique, 2000.

13 CAUNE, Jean, «La médiation culturelle: une construction du lien social ". Article en ligne, novembre 1999. <http:// w3.u-grenoble3.fr/les_enjeux/2000/Caune/index.php> (consulté le 26 décembre 2012).

14 SICARD, Monique, « Le musée acteur ». In. GAILLARD, Françoise et Daniel BOUGNOUX (dir.). Les cahiers de médiologie, no 11 - Communiquer/Transmettre. Paris: Gallimard, 2001, p. 166. <http://mediologie.org/cahiers-de-mediologie/> (consulté le 26 décembre 2012).
Il faut avoir le courage de chercher dans la nuit, ce qui est propre à notre époque et ne se donne pas à voir dans la clarté et le bruit du temps présent. "Seul peut se dire contemporain, celui qui ne se laisse pas aveugler par les lumières du siècle et parvient à saisir en elles, la part de l'ombre, leur sombre intimité ${ }^{15} »$. Il s'agit, selon le philosophe, de vivre sans regrets, ce "rendez-vous qu'on ne peut que manquer " ou, pour le dire autrement avec Georges DidiHuberman et à la suite de Pier Paolo Pasolini et de Walter Benjamin, «d'organiser le pessimisme ${ }^{16}$, de veiller aux lueurs fragiles et ténues des lucioles, signes, œuvres ou communautés en devenir, mais lueurs dans la nuit de notre temps.

\section{Une médiation de l'art par l'art?}

Les médiations que nous appelons de l'art par l'art (par les pratiques d'arts) nous semblent aller dans le sens d'une évolution du paradigme de la médiation qui s'est déplacé, en une quinzaine d'années, du pédagogique vers l'expérientiel. Cette évolution ouvre selon nous à une autonomie plus grande des sujets (médiateurs comme visiteurs) et place l'expérience esthétique comme le lieu à partir duquel engager une pratique créative et réflexive. Notons qu'il ne s'agit pas de célébrer une opposition pédagogie | expérience, mais plutôt de signaler un renversement, une (r)évolution : faire tout d'abord l'expérience de l'art, l'approcher par une pratique créative, puis partager, co-naître, savoir, critiquer, faire.

Les médiations de l'art par l'art proposées par les institutions font le plus souvent appel à des artistes ou à des collectifs renommés pour animer des ateliers de danse, d'écriture, de

15 AGAMBEN, Giorgio. Qu'est-ce que le contemporain? Paris: Payot et Rivages, coll. "Rivages poche / Petite Bibliothèque ", 2008, p. 21.

16 DIDI-HUBERMAN, Georges. Survivance des Lucioles. Paris : Minuit, 2009; PASOLINI, Pier Paolo. " Le vide du pouvoir en Italie " (dit aussi "L'article des lucioles "). Corriere della sera, 1er février 1975 ; BAUDRY, Marie. "Lucioles malgré tout. Comment "organiser le pessimisme" ?". Acta Fabula, vol. 11, no 1, 2010. <http://www.fabula.org/revue/ document5425.php> (consulté le 19 décembre 2012). 
musique, de lectures, organisant un face à face singulier avec une œuvre ${ }^{17}$. Les médiateurs se trouvent alors disséminés dans le groupe lors des activités d'atelier, assurant les rôles d'intermédiaires institutionnels, d'animateurs, de passeurs, de veilleurs. Selon Élisabeth Caillet, "Le médiateur n'est [...] pas celui qui enseigne, mais celui qui conduit vers ceux qui savent. Il se situe ainsi entre le savoir (scientifique ou culturel) ou le dispositif technique d'un côté et le public de l'autre ${ }^{18}$. " Les médiations de l'art par l'art sont répandues en musée ou en programmes hors les murs depuis le milieu des années 2000 et constituent, depuis, la part prisée des modalités de médiation par les publics, notamment scolaires. Elles ont ouvert la voie à d'autres inventions : nous allons maintenant nous interroger sur les enjeux médiologiques et relationnels d'un dispositif innovant de médiation de l'art par le design.

\section{Une médiation aidée par le design?}

\section{"Les jeunes ont la parole ", musée du Louvre 2012-2013}

Le programme que nous étudions a été proposé et mis en place par Joël Paubel pour l'édition 2012-2013 des nocturnes " Les Jeunes ont la parole " organisées par le (et au) musée du Louvre à Paris. Cet événement a lieu chaque année depuis 2004 et témoigne de la politique volontariste et expérimentale menée par l'établissement en direction des jeunes qui étaient trop peu nombreux à fréquenter les collections

17 Parmi ces expériences, citons celle menée au Grand Palais lors de l'exposition Monumenta 2008 avec un atelier de médiation par la danse en partenariat avec le Théâtre national de Chaillot autour de Promenade, œuvre monumentale en acier de Richard Serra. <http://archive.monumenta. com/2008> (consulté le 18 décembre 2012). 18 CAILLET, Élisabeth. "La médiation du design, pour une esthétique du quotidien ", article paru dans Place au design, inaccessible depuis la refonte du site Web de l'ENSCI (École nationale supérieure de création industrielle) Paris (n.d.). Élisabeth Caillet est responsable de l'action culturelle et des expositions au musée de l'Homme à Paris. Elle a publié plusieurs ouvrages sur la médiation culturelle, dont À l'approche du musée, la médiation culturelle. Lyon: Presses universitaires de Lyon, 1996.

19 Voir le site des JOP : <http://www.louvre.fr/programmejeunes-18-30-ans-du-musee-du-louvre> (consulté le 19 décembre 2012). du musée. Aujourd'hui, les moins de trente ans représentent près de la moitié des 8,5 millions de visiteurs annuels ${ }^{19}$.

La proposition de Joël Paubel a tenté le pari de réaliser des situations de médiation inédites de l'art par le design: 120 designers en formation dans cinq écoles de design - arts appliqués de Paris ${ }^{20}$ ont réalisé 120 objets de design qui par leur nature, leurs matériaux, leur fonction d'objet interrogent l'une des 120 œuvres d'art choisies par l'équipe dans différentes collections du musée.

Ces dispositifs de triangulation œuvres d'art patrimonial / objets de design contemporain / médiations ont lieu en situation au musée, accompagnés par 120 étudiants en médiation culturelle et histoire de l'art issus d'universités parisiennes partenaires ${ }^{21}$.

Les nocturnes se déroulent en deux temps: une première série de trois soirées ont eu lieu en novembre 2012 et une seconde série de trois soirées se tiendront en avril 2013. À la date de rédaction de cet article (début 2013, soit à l'intersection de ces deux périodes), le travail d'enquête a permis de réaliser l'observation et la documentation photographique de huit dispositifs composés chacun d'une œuvre, d'un objet de design et des discours croisés d'un médiateur ou une médiatrice et d'un ou une designer. Nous avons réalisé les entretiens sur place, en situation, auprès des designers et des médiateurs et du public utilisant les dispositifs

20 Les objets de design ont été réalisés par les étudiants du BTS (brevet de technicien supérieur) et du DSAA (diplôme supérieur d'arts appliqués) des lycées Jacques Prévert de Boulogne et Le Gué de Tresmes, des écoles supérieures d'arts appliqués de Paris (Duperré, Boulle et Estienne), sous la responsabilité de leurs professeurs: Dominique Robert, Carine Terrasson, Maëlle Chastanet, Anne-Marie Septfonds, Marielle Mathieu, Raphaël Lefeuvre, Michèle Mabille, Antoine Barnaud, Magali Fossier, Delphine Gauly et Élise Gourdy-Bléniat.

21 La Direction de la politique des publics et de l'éducation artistique du Louvre sollicite pour ce projet, avec Joël Paubel, les enseignants et les étudiants de médiation culturelle de l'ESPE de l'Université Cergy-Pontoise, du Département histoire de l'art de l'Université Paris Ouest Nanterre, des UFR (unités de formation et de recherche) Histoire et arts et Sciences de l'art de l'Université Paris I, d'Histoire de l'art de l'École du Louvre et de l'American University of Paris. 
proposés. Nos questions portaient pour les designers sur la genèse de la réalisation de leur objet de médiation et, à chacun (médiateur et designer), sur la façon dont la présence de cet objet modifie la relation de médiation et sur la manière dont chacun se positionne en situation de médiation. Nous ne pourrons pas ici faire une description et une étude détaillées de tous les objets mis à la disposition du public, car la recherche est en cours de réalisation.

Nous avons cependant à ce jour suffisamment de matériel d'observation pour commencer à interroger le dispositif de médiation mis en place au Louvre à travers l'analyse de deux objets que nous qualifierons de médiateurs. Nous tenterons également, dans un aller-retour réflexif-analytique, d'explorer les méthodologies qui semblent les plus pertinentes à adopter pour évaluer les propriétés des objets-médiateurs produits dans ce dispositif et, plus largement, pour mettre en perspective la grande boucle du réseau des objets hybrides mis en présence. La nature très singulière des types d'objets mis en présence (d'art et de design), le fait que les uns soient des œuvres patrimoniales riches d'une inscription historique et esthétique majeure, que les autres soient des propositions contemporaines d'objets techniques ou technologiques, nous poussent à nous interroger particulièrement sur les enjeux épistémologiques de notre enquête.

\section{De l'objet de design à l'objet médiateur}

Le pacte des JOP consiste en la mise à disposition du visiteur qui le souhaite d'un objet technique contemporain confié au musée par le designer. Cet objet peut être de nature très différente selon les cas : rouleau de papier imprimé, boîte d'échantillons, instrument, appareil optique, équipement audio, tablette numérique, installation interactive, etc.

22 Communiqué de presse du Louvre, op. cit.

23 Joel Paubel rapporte dans une interview recueillie au musée du Louvre le 7 décembre 2012: « les premières idées des étudiants en design étaient davantage tournées vers l'installation d'art contemporain, peut-être parce que celle-ci fait partie des formes d'interventions connues en lieu patrimonial, citons les objets de Murakami à Versailles par exemple ». Les normes drastiques du Louvre ont accompagné son conseil
Nos premières observations montrent que le visiteur cherche très vite à savoir comment s'en servir dans son approche de l'œuvre. Il comprend aussitôt qu'il se trouve au sein d'une médiation instrumentée, et ce, même sans consigne particulière. Le musée spécifie toutefois une série de questions dans un communiqué de presse: "Cette saison les "Jeunes ont la parole" interrogent notre regard sur l'œuvre. Comment la regarder? Quels sont nos points de vue? Sommes-nous attirés par un détail ou par la composition d'ensemble? Quel est l'impact de son environnement, de la salle où elle est exposée 22 ?"

En situation dans l'espace du musée, le visiteur différencie rapidement l'objet technique ${ }^{23}$ qui lui est confié, qu'il peut toucher, dont il peut se servir, de l'œuvre d'art exposée, qu'il ne peut pas toucher et qui n'a pas d'utilitéfonctionnelle. La convention culturelle implicite est suffisamment partagée pour que, dans l'enceinte du musée du Louvre, aucun doute ne soit permis quant à la différence de statut des objets mis en présence et que le schéma présenté dans l'illustration 2 pourra objectiver.

Nos premières observations mettent d'emblée en avant le caractère ludique de l'expérience par l'usage des objets de design proposés. Le public se presse avec curiosité et intérêt autour du visiteur qui manipule l'objet de design et tente d'en comprendre le fonctionnement. Celui-ci le tourne, le retourne, le détaille, pose quelques questions sur le mode d'emploi, le soupèse, puis s'en saisit pour appréhender l'œuvre. L'objet est la plupart du temps convoité par des personnes du public qui attendent leur tour, participant souvent à la discussion qui s'installe, particulièrement lorsque le visiteur-testeur se montre satisfait de

d'en rester à « la conception d'objets de taille modeste, précis, efficaces, en regard d'une œuvre ". Nous notons qu'il est resté quelque chose de la volonté initiale des étudiants: chaque objet de médiation, aussi modeste soit-il, porte bel et bien la trace de leurs choix subjectifs et se lit comme réponse pour une œuvre précise plutôt que pour une fonction générale, comme c'est souvent le cas en design, ce qui confère aux objets produits un statut très particulier. 


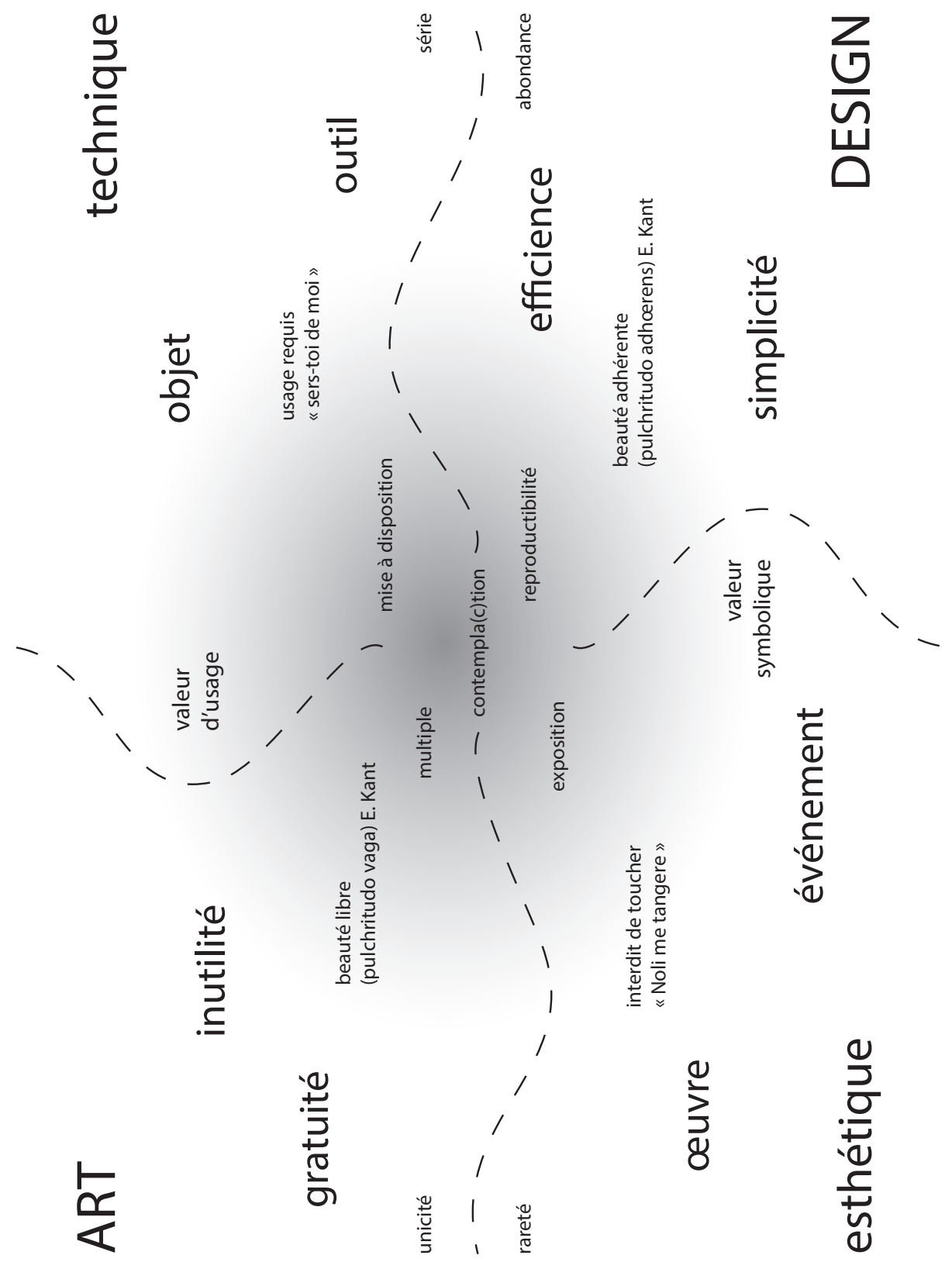

Illustration 2

Luc Dall'Armellina, « carte des relations objets de design / œuvres d'art ", 2013. 
ce que cet objet lui a permis de rendre visible, de dévoiler, d'une manière plus intime qu'un discours ne l'aurait fait.

Ici, pas (encore) de trame historique ou savante, juste un objet dans les mains et une question adressée au visiteur: "Que vois-tu?" Le discours ne précède pas, mais succède à l'expérience technique, kinesthésique, auditive, visuelle, cognitive et sensible faite par le visiteur aidé de l'objet de design. Les visiteurs interrogés sur ce renversement dans la médiation (expérimenter puis parler) disent très bien comment ils ne se taisent plus à cause de leur ignorance en histoire ou en esthétique, mais comment ils se mettent à parler grâce à l'expérience qu'ils viennent de faire à partir d'un objet de design encapsulant certaines fonctions médiatrices.

\section{Des objets aux fonctions médiatrices?}

Quelles sont-elles, ces fonctions médiatrices? sans doute, dans la poursuite de notre recherche, décrire très précisément les processus de design pour chacun des objets médiateurs créés. Nous pouvons à ce stade faire cette proposition: une fonction médiatrice est encapsulée dans un objet technique lorsque des qualités matérielles, fonctionnelles, ergonomiques, cognitives, esthétiques, émerge une affordance telle que dans l'usage de l'objet s'inventent des usages et que parle quelque chose comme une

24 SIMONDON, Gilbert. Du mode d'existence des objets techniques. Paris: Aubier, coll. « Res - L'invention philosophique ", 1958, 1969, 1989. Citons, par exemple, page 248 : "Par l'intermédiaire de l'objet technique se crée alors une relation interhumaine qui est le modèle de la transindividualité » et, plus loin: «L'objet qui sort de l'invention technique emporte avec lui quelque chose de l'être qui l'a produit, exprime de cet être ce qui est le moins attaché à un hic et nunc; on pourrait dire qu'il y a de la nature humaine dans l'être technique $[\ldots]$ ".

25 Voir par exemple la présentation de l'approche écologique de la perception visuelle de James J. Gibson dans TAMARA, Leonova. "L'approche écologique de la cognition sociale et son impact sur la conception des traits de personnalité ". L'année psychologique, vol. 104, no 2, 2004, p. 249-294. 26 AKRICH, Madeleine. "Technique et médiation ". Réseaux, vol. 11, no 60, 1993, p. 87-98. <http://www.persee.fr/ web/revues/home/prescript/article/reso_0751-7971_1993_ num_11_60_2368> (consulté le 26 décembre 2012).

subjectivité24. L'affordance est un concept central en design et particulièrement en regard de la dimension ergonomique d'un objet. Ce mot vient de la psychologie cognitive ${ }^{25}$ qui signale la capacité d'un système ou d'un produit à suggérer sa propre utilisation. En d'autres termes, on dira de cet objet qu'il est intuitif, c'est-à-dire qu'il ne nécessite pas de mode d'emploi pour son usage.

Si la médiation par l'objet technique n'est pas nouvelle pour les regards attentifs de la philosophie des techniques ${ }^{26}$ ou de la médiologie ${ }^{27}$, qu'elle a déjà pris des formes proches avec les questions de la muséologie en milieu scientifique et technique ${ }^{28}$, la médiation de l'art par le design se présente en musée patrimonial comme une forme originale, car elle s'y invite avec les modalités qui étaient jusqu'ici plutôt celles des musées des sciences et techniques. Monique Sicard rappelle en effet:

Dans les musées ne disposant pas de collections, le voir est sollicité de manière privilégiée: faire le tour d'une question, c'est exercer sa raison et son jugement. Ce regard-là n'est pas limité à l'exercice des yeux, mais il prend en compte la manipulation, l'expérience, l'interactivité ${ }^{29}$.

Voici très exactement ce que se proposent d'opérer la plupart des propositions faites par les designers engagés dans cette expérience de médiation aidée de l'objet technique auprès d'œuvres patrimoniales du musée du Louvre: organiser et scénariser des modalités

27 SICARD, Monique. "Eco-medio, la paire imparable ". In. MERZEAU, Louise (dir.). Les cahiers de médiologie, no 6, 1998 - Pourquoi des médiologues. <http://www.mediologie. org/cahiers-de-mediologie/06_mediologues/sicard.pdf > (consulté le 26 décembre 2012).

28 SICARD, Monique. "Ce que fait le musée... Science et art, les chemins du regard ». Publics et Musées, no 16, 1999, p. 41-53. <http://www.persee.fr/web/revues/home/prescript/article/pumus_1164-5385_1999_num_16_1_1140> (consulté le 26 décembre 2012). L'auteure montre dans ce texte comment les réalités institutionnelles des musées des arts et des sciences diffèrent jusqu'en matière de médiation et comment celles-ci révèlent un clivage des arts et des sciences mis en partage dans l'ère Malraux (1959), entre les ministères de la Culture d'une part et de l'Éducation nationale et/ou de la Recherche d'autre part.

29 SICARD, id., p. 45. 
d'appropriation médiées. «Schématiquement, les musées de science portent à la lumière ce qui doit être vu tandis que les musées d'art se soucient de ce qui doit être montré. Les premiers sont fondamentalement concernés par une transmission immédiate, une éducation, une explication. Les seconds par une transmission différée ${ }^{30}$."

Il se pourrait pourtant qu'une médiation de l'art par le design renverse les partitions temporelles relevées notamment par Sicard en 1999. En analyser ici les raisons nous emmènerait trop loin, mais nous pouvons déjà mettre au crédit de ces changements un rapport contemporain à l'objet technique (et, plus encore, numérique) généralisé et bien plus établi dans nos pratiques qu'il ne l'était il y a quinze ans, et ce, à tous les niveaux de notre vie.

\section{Deux dispositifs objet de design/œuvre d'art/médiateurs}

\section{Conic, ou le révélateur de perspectives}

Dans ce dispositif, la médiatrice est designer en formation au Lycée du Gué à Tresmes en BTS design d'espace. Avec deux collègues ${ }^{31}$, elle a conçu le dispositif optique Conic que l'on voit, sur l'illustration 3, utilisé par une enfant: c'est un cône de 25 centimètres de diamètre sur 40 centimètres de haut, réalisé en fines tiges de métal soudées que l'on tient grâce à une poignée, pointé vers l'objet à voir comme avec tout appareil à visée, la partie pointue du cône dirigée vers le tableau: La Vierge et l'Enfant entourés de deux anges, sainte Rose et sainte Catherine d'Alexandrie, peint vers 1490-1495'32. Le dispositif proposé ici permet de suivre les lignes de fuite de cette œuvre structurée selon les principes de la perspective, en faisant correspondre lignes du tableau et filins de laine ajustables sur l'objet médiateur.
Une fois l'expérience effectuée sur le tableau du Perugin, le visiteur fera souvent seul l'exercice de se détourner de la peinture et de regarder au loin, vers le point de fuite de la grande galerie dans laquelle il se trouve, transformant une proposition de médiation culturelle en usage d'un appareil universel de vision et de mesure de la perspective.

Ce qui pourrait être vu comme un détournement d'usage par rapport à la consigne de médiation constitue peut-être le signe de l'appropriation complète d'un dispositif offrant une modalité du voir simple, originale et particulièrement pertinente, même si bien sûr l'approche d'une œuvre ne saurait s'y limiter.

Nous nous interrogions plus haut sur ce que recèlent les fonctions médiatrices d'un objet de design. Nous pourrions dire dans le cas présent qu'elles consistent en l'organisation d'une modalité du voir qui met en scène et en condition d'expérimentation, la déconstruction géométrique de la vue en perspective amenant le visiteur à objectiver cette notion qui sans cela resterait abstraite.

Ce qu'un discours soutiendrait intellectuellement dans le temps et la démonstration, un objet simple et ludique le met en application dans l'instant de l'expérience. Mais nous pourrions tout aussi bien dire que ce que l'objet médiateur met à la disposition du visiteur, c'est un instrument qui l'amène à l'expérience d'appréhender différents types de perspective (linéaire, axonométrique, inversée) en même temps qu'il peut l'attirer au centre des questions esthétiques du point de vue: de dieu ou de l'infini, du peintre ou du regardeur, etc. C'est depuis la richesse de cette expérience, dont il ressort assuré de sa capacité à éprouver, à lire et à interpréter, que le visiteur, adulte ou enfant, peut poser des questions, maintenant curieuses, au médiateur qui n'aura non seulement rien perdu dans sa relation avec le visiteur, mais aura

32 Ce tableau est attribué à Pietro di Cristoforo Vannucci, dit Perugin, Città della Pieve, vers 1450 - Fontignano (Pérouse), 1523. Situation au Louvre: Grande Galerie, aile Denon, étage 1 , salle 5 .
30 Id., p. 44.

31 Marlène Gaillard (designer et médiatrice interviewée), avec Manon Legal et Lylia Ibouri. 


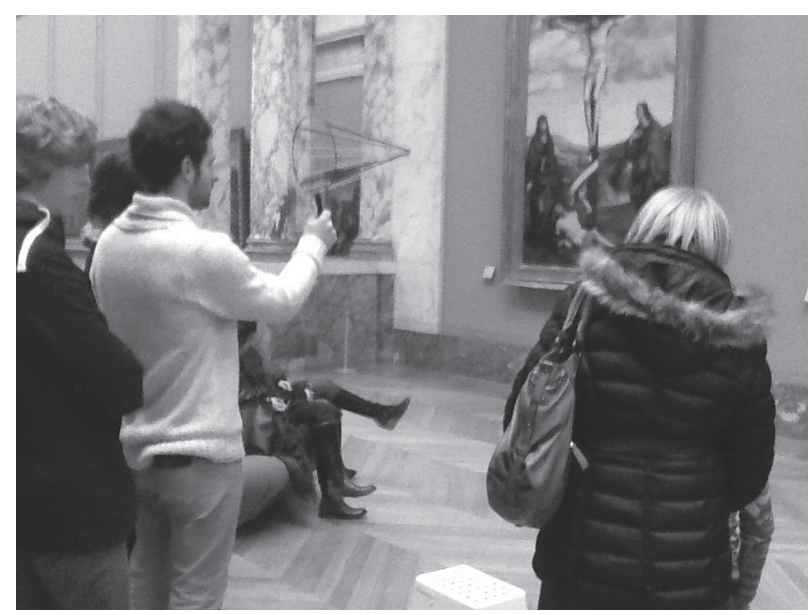

Dispositif « Conic » de Marlène Gaillard, Manon Legal, Lylia Ibouri en situation d'usage.

[Photo: Luc Dall'Armellina, musée du Louvre, 2012]
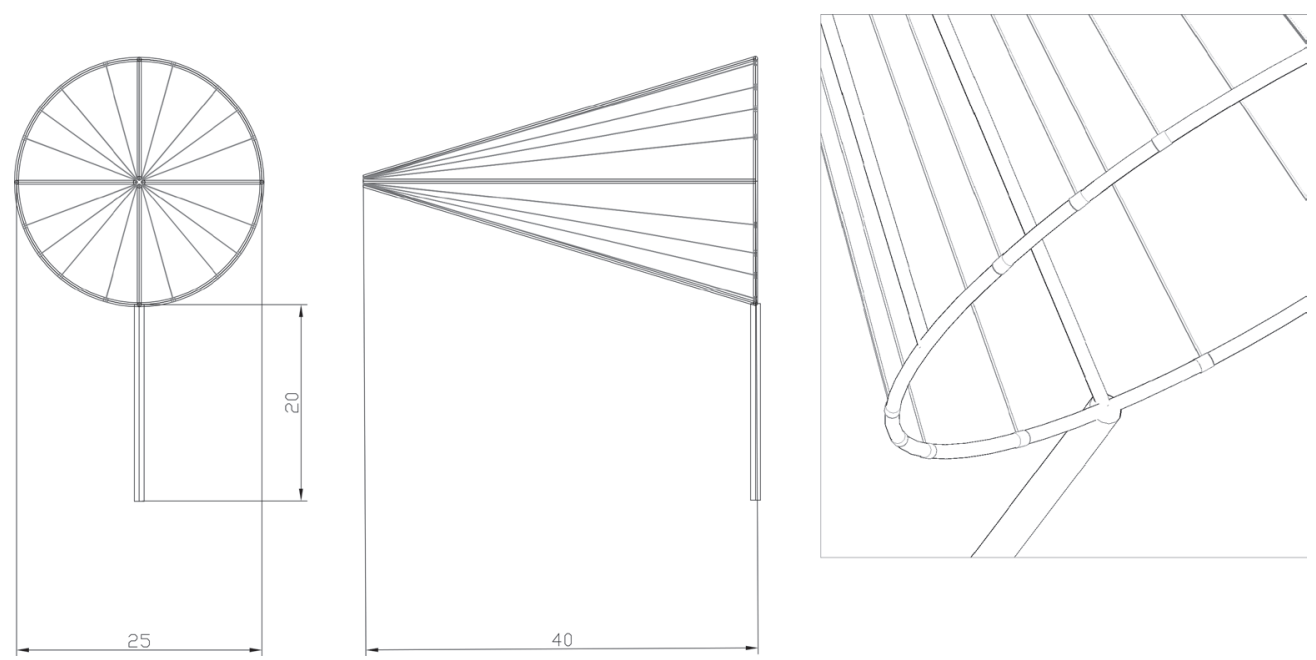

\section{Illustration 3}

Marlène Gaillard, Manon Legal, Lylia Ibouri, géométraux de « conic », 2012. 
gagné d'engager un échange plus informé et exigeant, à partir de la connaissance active et valorisante que vient de faire son interlocuteur.

En permettant au visiteur de faire une expérience publique de principes visuels fondateurs, c'est aussi au specta(c)te ${ }^{33}$ qu'il est invité, c'est-à-dire à la mise en spectacle de la captation et de l'orientation de son regard instrumenté par l'objet-médiateur, ceci sous le regard des autres visiteurs, créant une situation de partage spontanée (que nous avons constatée fréquemment).

Cette mise en spectacle et la mise en scène qui la sous-tend, Sicard l'avait repérée pour le musée des sciences: « Ces quatre figures muséographiques - du conserver, du circuler, du manipuler, du mettre en scène - ne sont certes ni étanches ni exclusives mais elles peuvent servir de guide à toute analyse du musée comme matérialisation d'un regard ${ }^{34}$." Matérialisation, mise en scène et ici instrumentation du regard sont autant de facettes d'un dispositif de médiation qui montre en effet qu'en appareillant le regard, c'est au risque du spectacle que le musée expose ses visiteurs. Sans doute aucune des figures muséographiques citées plus haut (conserver, circuler, manipuler, mettre en scène) n'est à l'abri des excès, mais n'est-ce pas l'enjeu de toute approche muséale que de trouver la mesure adéquate de cette matérialisation du regard?

La médiologie avait dans le projet de Régis Debray $^{35}$ cette façon singulière d'articuler deux temporalités que sont les temps courts et fonctionnels de la communication à ceux, longs et structurels, de la transmission. «Dans médiologie, "médio" ne dit pas média ni médium, mais médiations, soit l'ensemble dynamique

33 Jean-Louis WEISSBERG est l'inventeur de ce néologisme apparu dans son livre Présences à distance. Déplacement virtuel et réseaux numériques: Pourquoi nous ne croyons plus la télévision. Paris: L'Harmattan, 1999. Ce néologisme est issu de l'analyse des hypermédias et du jeu vidéo, mais la situation scopique instrumentée et publique décrite ici semble relever d'une situation très proche, à ceci près que le dispositif technique n'est pas numérique mais optique.

34 SICARD, Ce que fait le musée..., op. cit., p. 45.

35 DEBRAY, Régis. Cours de médiologie générale. Paris:

Gallimard, 1992, p. 17. des procédures et corps intermédiaires qui s'interposent entre une production de signes et une production d'événements ${ }^{36}$. "Une telle approche a sa place dans notre étude, car ce n'est pas le hasard qui a conduit Monique Sicard à poser les questions de la fabrication du regard en musée ni François Dagognet à rappeler les enjeux de prendre le temps d'établir des rapports aux objets culturels: "Nous revenons ainsi à une thèse que nous avons défendue, une conception de la culture intussusceptive (intus, à l'intérieur et susceptio, l'action de prendre) ce qui suppose le temps d'incorporer, et refuse, du même coup, l'hyper-consommation ${ }^{37}$."

L'approche médiologique que Debray n'a jamais souhaité qualifier de discipline - et qu'on pourrait même avec Yves Jeanneret qualifier d'anti-discipline ${ }^{38}$-, peut-être pour la protéger des effets de système, devrait se montrer capable de révéler les enjeux des formes de transmission propres à la médiation culturelle, et ceci dans toutes ses phases. Son approche ouverte et transdisciplinaire nous intéresse par sa capacité à inventer un éclairage en toute indépendance:

S'il devenait un spécialiste ès médiologies, le médiologue perdrait en revanche son âme (et son corps), car il perdrait sa faculté précieuse de chercher à superposer (société du spectacle et machineries, route et religions, réseaux techniques et Nation, croyance et papier, bicyclette et culture...). Il ne parlerait plus que théorèmes ou statistiques, métaphores ou questionnaires. Il renoncerait à l'inconfort de l'entre-deux, et basculerait d'un seul côté de ces traits d'union qu'il s'est donnés pour lieux de transit:

36 DEBRAY, Régis. Manifeste médiologique. Paris: Gallimard, 1994, p. 29. 37 DAGOGNET, François. "Incorporer ». In. MERZEAU, Louise (dir.). Les cahiers de médiologie, no 6 - Pourquoi des médiologues. Paris: Gallimard, 1998, p. 184. 38 JEANNERET, Yves. "La médiologie de Régis Debray ". Communication et langages, no 104, 2e trimestre 1995, p. 4-19. 
technique-culture, pensée-matière, opinion-institution, faire-croire... Bref, la médiologie doit cultiver son impureté pour garantir son efficacité ${ }^{39}$.

À travers le dispositif " Conic " examiné ici très sommairement, il nous semble que l'approche médiologique, qui a permis d'isoler finement les dimensions macro des dispositifs institutionnels ${ }^{40}$ comme de mesurer la portée micro des objets-médiateurs, puisse céder la place à une approche plus résolument systémique, écologique. Nous entendons par là une approche qui puisse saisir, dans le même mouvement, les humains et les objets, les mythes et les savoirs, les arts et les techniques. N'est-ce pas dans la direction d'une interdisciplinarité assumée qu'il faudra chercher une méthode pour saisir nos objets complexes?

Il nous semble que la médiologie, qui a surtout développé ses approches dans l'interdiscipline de la sémiologie et des sciences de l'information et de la communication, puisse aujourd'hui prolonger son investigation des " réseaux " vers la sociologie de la traduction dite aussi théorie de l'acteur réseau (ANT / actor-network theory). Ancrée en anthropologie et en philosophie des techniques, celle-ci semble en effet pouvoir rendre compte des multiples modes d'existence des objets, expériences et singularités mis en présence dans notre situation de médiation de l'art par l'objet de design. Nous le verrons dans l'exemple suivant.

\section{Le tableau magique ou le comble du manque}

Dans le projet de tableau magique conçu et réalisé par deux étudiantes ${ }^{41}$ en DSAA création-conception multimédia du Lycée Jacques Prévert de Boulogne, celles-ci proposent au visiteur de dessiner les parties manquantes de la statue Aphrodite accroupie $e^{42}$ œuvre romaine

39 MERZEAU, Louise. "Ceci ne tuera pas cela". In. MERZEAU, Louise (dir.). Les cahiers de médiologie, no 6 Pourquoi des médiologues. Paris: Gallimard, 1998, p. 28. 40 SICARD, Ce que fait le musée..., op. cit.

41 Mathilde François et Hélène Taïeb. d'époque impériale (Ier-IIe siècles après J.-C.). Elles lui demandent de s'assoir à quelques mètres de l'œuvre, derrière un petit châssis en bois de leur fabrication, encadrant un écran de plexiglass ${ }^{\mathrm{TM}}$ transparent à travers lequel le visiteur-dessinateur voit l'œuvre et sur lequel il va pouvoir dessiner ses parties manquantes... mais sans voir lui-même ce qu'il a dessiné.

Le dessin est produit à l'aveugle sur « l'ardoise magique ", qui semble sans mémoire, voire sans effets. Mais mémoire il y a pourtant... Elle a lieu en coulisses, s'affiche et s'enregistre sur l'ordinateur placé derrière le visiteur-dessinateur. Effets il y a aussi, puisque le dispositif reçoit par ondes infrarouges chaque coordonnée, pression et direction des mouvements du dessin produit par le stylet numérique.

Les dessins sont archivés anonymement par date et font l'objet d'une collecte documentée sur le site Internet dédié au projet ${ }^{43}$. Ce dispositif consiste donc à proposer - en suspendant tout signal de retour d'action pour le dessinateur - un geste d'augmentation par le dessin pour compléter la vue d'une œuvre sculptée amputée (en partie) de sa tête et de ses pieds.

Les deux auteures déclarent sur leur site, sous l'onglet " À propos du projet ":

C'est la notion de manque qui a motivé notre projet; en voyant les visiteurs du Louvre mettre leur tête à la place de l'Aphrodite et se photographier, nous avons voulu replacer cette pratique dans une intervention réelle sur l'œuvre. L'imagination a une place primordiale, et c'est grâce au tableau interactif que la reconstitution est possible. Grâce à toutes vos interventions, parviendrons-nous à reconstituer les parties manquantes de la sculpture? Visitez notre blog et découvrez par vous-même les propositions des visiteurs des soirées.

42 Situation au Louvre: aile Sully, rez-de-chaussée, salle d'art grec classique et hellénistique, salle 1. <http://www. louvre.fr/oeuvre-notices/aphrodite-accroupie> (consulté le 11 janvier 2013).

43 Voir:<http://cargocollective.com/aphroditeaccroupie> et le site d'Hélène Taïeb : <http://www.helenetaieb.fr/> (consultés le 26 décembre 2012). 
En privant le visiteur de tout retour sur ce qu'il vient de dessiner, les deux designers l'obligent à produire un geste différent. Pourquoi ? Lorsqu'il voit ce qu'il produit, un dessinateur non expert opère le plus souvent par touches successives, réparatrices, réorientatrices du trait à donner à telle forme, il est dans une construction lente qui autorise le repentir, jusqu'à son recouvrement. Mais lorsqu'il ne voit pas ce qu'il produit, il doit résoudre son dessin en un nombre très restreint de traits: ceux mémorisables sans artéfact. Dans cette opération rapide, il déroule une action guidée par sa seule vision directe et ne peut garder en mémoire tous les détails formant le dessin déjà réalisé, mais sans traces. Cette contrainte forte induit / produit une stylistique de l'économie du geste que l'on trouve par exemple dans les dessins d'Henri Matisse. Ce dispositif demande au dessinateur, par son jeu de contraintes, d'aller vite et à l'essentiel, ce que la plupart des images produites et exposées sur le site Web du projet reflètent très bien. Le visiteur fait ici une expérience créative et, si l'on peut dire, " réparatrice ", en ce sens qu'il met en actes depuis son imaginaire la reconstitution des fragments manquants d'un corps, d'une pose, d'une scène initiale qui échappe et échappera nécessairement à tous. Il fait cette expérience, placé lui-même en situation de manque, ne sachant s'il a réussi ou non à reconstruire une image complète. Il ne verra qu'après coup, et seulement s'il le souhaite, le dessin qu'il a réalisé. Ici aussi, une médiation plus classique aura toute sa place, non plus par défaut avant toute chose et sur le mode du savoir, mais à partir des sensations, frustrations et questions issues d'une expérience sensible et inédite de dessin. Nous pourrions penser qu'une approche esthétique serait ici en mesure de soutenir et de prolonger l'expérience du visiteur dans une direction différente que l'histoire de l'art ne le ferait, sans doute d'une manière favorable aux

44 Voir le site de Johnny Chung Lee : <http://johnnylee.net/ projects/wii> (consulté le 27 décembre 2012).

45 LATOUR, Bruno. Changer de société - Refaire de la sociologie. Paris: La Découverte, coll. "Armillaire ", 2006, p. 217.

Le chapitre dont est tirée cette citation est le dialogue entre un professeur et un étudiant sur "l'efficience " de l'ANT.

46 LATOUR, Bruno. Enquête sur les modes d'existence. Une anthropologie des Modernes. Paris: La Découverte, 2012. dimensions critiques. Il y a là de quoi motiver l'élargissement des compétences pour l'exercice de la médiation culturelle à l'heure des objets-médiateurs complexes.

Si nous sommes tentés ici d'interroger le dispositif technique mis en place: captation des mouvements du dessin par le détournement ${ }^{44}$ d'une manette de console de jeu Nintendo $\mathrm{Wii}^{\mathrm{TM}}$ et son interfaçage avec un stylet et un ordinateur, c'est en suivant cette idée qui vient de la sociologie de la traduction (ANT) et qui consiste à penser les objets comme des hybrides nature-culture, technique-esthétique, pris dans un réseau hétérogène dans lequel les faits comme les objets, les idées comme les sujets, les collectifs comme les institutions sont des acteurs / actants. Cette approche en refusant la séparation de l'humain et du non-humain nécessite un travail de traduction rendant les connexions du réseau intelligibles, car « il n'y a jamais d'information, mais seulement des transformations, ou de la traduction si vous préfére ${ }^{45}$ ". Au centre du dispositif d'analyse de l'ANT, la controverse est une pratique d'auscultation et de mise en évidence des problèmes critiques posés. Cette position difficile est tenue par quelques chercheurs depuis les années 1980 autour de Bruno Latour, Madeleine Akrich et Michel Callon, du Centre de sociologie de l'innovation de Mines Paris Tech, mais presque exclusivement dans le domaine des sciences et des techniques.

La médiation culturelle des arts par le design ne pourrait-elle pas élaborer l'analyse de ses pratiques à l'aide des approches conceptuelles de l'acteur-réseau? Les travaux les plus récents de Latour ${ }^{46}$ montrent depuis 2001 une extension de ses activités critiques aux arts ${ }^{47}$ et, en 2007, au MédiaLab de Sciences Po Paris, au champ de l'analyse cartographique des controverses dans lequel le design d'information joue un

47 Voir les sites du ZKM (Zentrum für Kunst und Medientechnologie Karlsruhe / Center for Art and Media of Karlsruhe) : <http://www.zkm.de/iconoclash/> et de l'exposition Iconoclash: <http://www.iconoclash.de> (consultés le 27 décembre 2012). 
rôle important ${ }^{48}$. Mais de quelle approche analytique et critique la médiation de l'art par le design a-t-elle besoin? D'une part pour continuer d'inventer sa pratique, et d'autre part pour évaluer la portée de ses actions? Et pour quelle production esthétique et politique? Peut-elle se limiter à une seule méthode ? Peut-elle en hybrider plusieurs, bricoler l'agencement dont elle a besoin? L'ANT n'est pas une panacée, mais nous apparaît plutôt comme une machine conceptuelle qui tente de dépasser les limites de l'approche sociologique " sociale " en s'attachant à la qualité de la description des événements laissant toute leur place aux acteurs, se gardant de toute surimposition théorique. "Il n'y a pas d'outils, pas de moyens. Un texte a une épaisseur. Ça c'est vraiment un précepte de base de l'ANT ${ }^{49}$."

\section{Pour recommencer}

L'approche méthodologique de l'acteur-réseau sera-t-elle à même de saisir la complexité, dans visiteur de nouvelles dispositions pour le dialogue, avec le médiateur, mais élargissant souvent le cercle aux autres visiteurs présents.

\section{La présence et l'usage d'objets techniques} médiateurs face aux ouvres ne va cependant pas toujours de soi. Dans le meilleur des cas, ceux-ci encapsulent des fonctions médiatrices et proposent un appareillage cognitif de l'attention (du regard, du geste, de la mémoire) et renouvellent les modalités de la relation visiteur-œuvre-médiateur, mais sont-ils à même d'ouvrir ou de faciliter l'expérience esthétique? Si non, pourquoi? Si oui, celle-ci est-elle partageable? De quelle manière? C'est ce que la poursuite de notre investigation pourra mettre en lumière.

Si quelques dispositifs produits par les étudiants de design ont déjà fait preuve de leur pertinence lors de la première partie des nocturnes du Louvre - et j'espère leur avoir rendu justice -, la méthode pour les dire sans les trahir aura à inventer sa voie, sans réduire la complexité des objets, faits et situations, acteurs, choses et réseaux. Elle aura aussi à en éclairer les facettes brillantes, comme celles exposées à la nuit. techno-cognitifs des acteurs (objets et sujets) médiateurs, des relations éthico-esthétiques qu'ils nouent, des dimensions économicopolitiques de la médiation muséale, et des ruses, arts et manières de faire des visiteurs? C'est une tâche qui nous semble, dans l'esprit de son approche, ouverte, attentive aux faits, dispositifs, actes et paroles de tous les acteurs, humains et non humains, qui composent le « réseau » de cette médiation.

Nous avons pu montrer dans cet article comment la médiation par le savoir s'est muée dans cette édition des "Jeunes ont la parole " en médiation par l'expérience, créant chez le 
Luc Dall'Armellina, translated by Allana Carlyle

\section{Design improving museum interpretation?}

Professional museum interpretation is the practice of accompanying the public on an aesthetic and analytical cultural experience of contemporary and patrimonial works of art. With this definition, museum interpretation is a challenging concept, caught between the inevitable modern habitus of cultural industries (consumption, limited attention spans) and the constant risk of the removal of the visitor's perceptive experience with art due to an over-talkative interpreter.

Luckily, museum interpretation has the power to create an environment that is not only based on knowledge and education, but which also looks to establish a creative relationship between the art and the museum visitor. Interpretation of art through the practice of the arts enters into this category. Institutions often call upon artists or other reputable groups to facilitate dance, writing, music or reading workshops in order to offer a unique encounter with art work.

In this article, we will explore the mediological and aesthetic aspects of an innovative plan for museum interpretation through design, which will be presented within the framework of the 2012-2013 edition of the "Nocturnes: Les jeunes ont la parole", organized by the Louvre Museum in Paris. Based on a proposal by Joël Paubel (artist, instructor at IUFM-Université Cergy-Pontoise), 120 young designers studying at five different schools of applied arts and design in Paris will create 120 design objects. These objects, due to their nature, material and function, will facilitate the examination of one of the 120 chosen art works at the museum. The meeting of art and design as well as it's interpretation will take place in the museum along with 120 students studying cultural interpretation at various Parisian universities.

The primary research topics examined in this article were studied prior to the presentation of "Nocturnes" which took place in November 2012 and April 2013. These topics are as follows:

- The description of a museum interpretation plan as it exists in a relational and complex way between objects, works of art and people from different cultures

- A reflection on the methodology used to analyze this unique experience in its different dimensions (interpretation, mediology, art critique, psychoanalysis, sociology of translation)

- The aesthetic aspects of an interpretation plan which leads visitors to explore the perceptive relationship between art and an object of contemporary industrial design

In such cases, is it necessary to appeal to the sociology of translation currently existing in the arts? The environment is familiar (laboratories, scientific procedures, controversy), but can it be adapted to the culture of art and design? And at what price? How is this made possible without omitting the discourse and unique characteristics of its players 
which make up complex contemporary aesthetics? What role is given to psychoanalytical approaches (from D. Winnicott to S. Tisseron) while highlighting the symbolic and cognitive dimensions of an individual's relationship with objects?

According to it's creator, the museum interpretation plan studied in this article has the goal of delaying the interpreter's intervention in order to create a more complete interpretation relationship. An object of design is placed in front of an art work. Due to it's specific placement, it brings into question it's function, materials, usability, engineering and basic identity as an industrial object. The designer and the museum interpreter are available to provide a discursive and unequivocal interpretation. In this case, a relationship is being established between five participants; a piece of art, a contemporary design piece, a museum interpreter, a designer and a visitor. The culture of arts and the culture of technical objects allow for some productive exchanges, but what is put at stake with this risky and improbable encounter? 